\title{
Perfil epidemiológico da Hanseníase no estado do Maranhão de 2018 a 2020
}

\author{
Epidemiological profile of Leprosy in the state of Maranhão from 2018 to 2020
}

Perfil epidemiológico de la Lepra en el estado de Maranhão de 2018 a 2020

Luciana Helenna Garces Anjos ORCID: https://orcid.org/0000-0002-1579-6588 Universidade Ceuma, Brasil E-mail: helennalu10@gmail.com

Sione Macedo da Cunha

ORCID: https://orcid.org/0000-0001-6422-4087 Universidade Ceuma, Brasil

E-mail: smdacunha@hotmail.com

Gardênia Monteiro Batista

ORCID: https://orcid.org/0000-0002-1874-5225 Universidade Ceuma, Brasil

E-mail: gardenia_mc@ hotmail.com

Taciana Mirely Maciel Higino ORCID: https://orcid.org/0000-0001-6704-2393 Fundação Altino Ventura, Brasil

E-mail: tacianahigino@gmail.com

Débora Carolina Pinto de Souza ORCID: https://orcid.org/0000-0001-6759-8412 Universidade Ceuma, Brasil

E-mail: deboracarolinapinto@gmail.com

Amanda Silva dos Santos Aliança ORCID: https://orcid.org/0000-0003-1412-9895 Universidade Ceuma, Brasil E-mail: amandaalianca2@gmail.com

\begin{abstract}
Resumo
Este estudo teve como objetivo analisar o perfil epidemiológico da Hanseníase no estado do Maranhão. Estudo transversal de caráter retrospectivo dos casos de hanseníase nos anos de 2018 a 2020 no estado do Maranhão, região nordeste do Brasil. Os dados foram obtidos por meio de consultas à base de dados do Sistema de Informações de Agravos de Notificação (SINAN), disponibilizados no Departamento de Informática do Sistema Único de Saúde (DATASUS). As variáveis utilizadas no estudo foram: macrorregiões de saúde, sexo, escolaridade e forma clínica. Entre os anos 2018 a 2020, de acordo com o DATASUS, foram notificados 10.776 casos de hanseníase no Maranhão, com média de 3592 casos/ano. No ano de 2018 foram notificados 4.195 casos em contraposição ao ano de 2020 com 2.316 casos, sendo o menor número de casos nos últimos 3 anos. No presente estudo foi observado que a hanseníase foi predominante no sexo masculino, na macrorregião Norte, nas pessoas da raça parda, em pessoas com baixo grau de escolaridade e a forma clínica mais comum foi a dimorfa. As condições sociais e vulnerabilidade associam a hanseníase a situações precárias de higiene e baixo nível econômico, levando a dificuldade de acesso à saúde. Espera-se, portanto, que os órgãos estaduais e municipais de saúde, promovam ao acesso a informações de saúde para população afetada, ações que promovam melhora dos indicadores de controle da doença no Maranhão, visando aperfeiçoar os aspectos relacionados à educação e saúde, tendo maior atenção ao público masculino, para que o tratamento da doença ocorra de forma eficiente.
\end{abstract}

Palavras-chave: Perfil epidemiológico; Hanseníase; Maranhão.

\begin{abstract}
This study aimed to analyze the epidemiological profile of leprosy in the state of Maranhão. Retrospective crosssectional study of leprosy cases from 2018 to 2020 in the state of Maranhão, northeastern Brazil. Data were obtained through consultations in the database of the Information System for Notifiable Diseases (SINAN), available at the Information Technology Department of the Unified Health System (DATASUS). The variables used in the study were: health macroregions, gender, education and clinical form. Between 2018 and 2020, according to DATASUS, 10,776 cases of leprosy were reported in Maranhão, with an average of 3592 cases/year. In 2018, 4,195 cases were reported, compared to 2020 with 2,316 cases, with the lowest number of cases in the last 3 years. In the present study, it was observed that leprosy was predominant in males, in the Northern macro-region, in people of the mixed race, in people
\end{abstract}


with low educational level, and the most common clinical form was borderline. Social conditions and vulnerability associate leprosy with precarious situations of hygiene and low economic status, leading to difficulty in accessing health care. It is expected, therefore, that state and municipal health agencies promote access to health information for the affected population, actions that promote improvement of disease control indicators in Maranhão, aiming to improve aspects related to education and health, having greater attention to the male public, so that the treatment of the disease occurs efficiently.

Keywords: Epidemiological profile; Leprosy; Maranhão.

\section{Resumen}

Objetivo: este estudio tuvo como objetivo analizar el perfil epidemiológico de la lepra en el estado de Maranhão. Metodología: estudio transversal retrospectivo de casos de lepra de 2018 a 2020 en el estado de Maranhão, noreste de Brasil. Los datos se obtuvieron a través de consultas en la base de datos del Sistema de Información de Enfermedades Notificables (SINAN), disponible en el Departamento de Tecnología de la Información del Sistema Único de Salud (DATASUS). Las variables utilizadas en el estudio fueron: macrorregiones de salud, género, educación y forma clínica. Resultados: Entre 2018 y 2020, según DATASUS, se notificaron 10.776 casos de lepra en Maranhão, con un promedio de 3592 casos / año. En 2018 se notificaron 4.195 casos, en comparación con 2020 con 2.316 casos, con el menor número de casos en los últimos 3 años. En el presente estudio se observó que la lepra fue predominante en varones, en la macrorregión norte, en mestizos, en personas con bajo nivel educativo, y la forma clínica más común fue borderline. Conclusión: Las condiciones sociales y la vulnerabilidad asocian la lepra con situaciones precarias de higiene y bajo nivel económico, lo que dificulta el acceso a la atención de salud. Se espera, por lo tanto, que las agencias de salud estatales y municipales promuevan el acceso a la información de salud para la población afectada, acciones que promuevan la mejora de los indicadores de control de enfermedades en Maranhão, con el objetivo de mejorar los aspectos relacionados con la educación y la salud, teniendo una mayor atención al público masculino. , para que el tratamiento de la enfermedad sea eficaz.

Palabras clave: Perfil epidemiológico; Lepra; Maranhão.

\section{Introdução}

As doenças negligenciadas (DNs) são condições infecciosas altamente prevalentes, marcadas pelo alto grau de morbidade, porém mortalidade relativamente baixa. Apesar de não ser exclusivas de países subdesenvolvidos, despertam pouco atrativo financeiro pela grande indústria farmacêutica, já que não atingem o grande mercado consumidor que são os países desenvolvidos anofelino-vetor (de Azevedo et al., 2020).

A hanseníase é uma doença infecciosa crônica considerada uma doença negligenciada. Ela é causada pelo bacilo Mycobacterium leprae, também chamado bacilo de Hansen, que é um parasita intracelular obrigatório, com afinidade por células da pele e por células dos nervos periféricos, que se abriga no organismo da pessoa contaminada (de Carvalho el al., 2018).

A doença caracteriza-se pelo acometimento dermatoneurológico e, apesar de curável e dos esforços de órgãos governamentais nos últimos anos por meio de políticas públicas, representa ainda um grande problema para a saúde pública no mundo e no Brasil. A transmissão da hanseníase ocorre por meio do contato prolongado e íntimo entre indivíduos susceptíveis ou predispostos geneticamente e pacientes multibacilares não tratados, da inalação de microrganismos eliminados pelas vias áreas superiores ou, em menor proporção, do contato direto. A mucosa nasal corresponde à principal via de entrada do bacilo. (de Almeida et al., 2020).

O diagnóstico é essencialmente clínico e epidemiológico. Não há teste laboratorial sensível e específico para concretizar a hanseníase. As lesões suspeitas de hanseníase devem ser estudadas por meio de exame físico dermatológico e neural, por profissionais aptos a realizar as condutas, por ser uma doença. No Brasil, a baciloscopia do esfregaço dérmico, quando disponível, deve ser utilizada como exame complementar para a classificação dos casos em Paucibacilares (PB) ou Multibacilares (MB) (Anchieta et al., 2019).

O tratamento é feito por um conjunto de antibióticos denominado poliquimioterapia (PQT), com o intuito de eliminar a bactéria do organismo adotada pela OMS a nível mundial. Após o diagnóstico, deve-se classificar o paciente hansênico para iniciar o esquema de terapia específico para o indivíduo. Dessa forma, para os PB utiliza-se o esquema de rifampicina, uma dose mensal de 600 mg, supervisionado na atenção básica de saúde, dapsona, uma dose mensal supervisionada e uma dose diária 
autoadministrada. Nesse caso, o tratamento dura 6 meses. Já a MB utiliza as mesmas doses da PB associando a clofazimina, uma dose mensal de $300 \mathrm{mg}$ com administração supervisionada e uma dose diária de 50 mg autoadministrada, sendo a duração do tratamento de 12 meses (de Carvalho el al., 2018).

A melhor forma de prevenção e controle da hanseníase é por meio do diagnóstico e detecção da infecção, triagem dos contatos familiares e tratamento precoce (Anchieta et al., 2019). Tendo em vista tal impacto, o projeto visa traçar um perfil epidemiológico da hanseníase no estado do Maranhão nos anos de 2018 a 2020.

\section{Metodologia}

Trata-se de um estudo transversal de caráter retrospectivo dos casos de hanseníase nos anos de 2018 a 2020 no estado do Maranhão, região nordeste do Brasil. Os dados foram obtidos por meio de consultas a base de dados do Sistema de Informações de Agravos de Notificação (SINAN), disponibilizados no Departamento de Informática do Sistema Único de Saúde (DATASUS). As variáveis utilizadas no estudo foram: macrorregiões de saúde, sexo, escolaridade e forma clínica.

Para tabulação e análise dos dados foram utilizados os programas Tabwin 3.6 e o Microsoft Office Excel 2010. Os dados foram analisados mediante estatísticas descritivas (frequências absolutas, gráficos e tabela).

\section{Resultados e Discussão}

Entre os anos 2018 a 2020, de acordo com o DATASUS, foram notificados 10.776 casos de hanseníase no Maranhão, com média de 3592 casos/ano. No ano de 2018 foram notificados 4.195 casos em contraposição ao ano de 2020 com 2.316 casos, sendo o menor número de casos nos últimos 3 anos (Figura 1).

Segundo Ferreira (2021), o impacto da pandemia e o efeito da suspensão dos atendimentos dermatológicos em função da pandemia da COVID-19, houve redução das atividades eletivas, gerando redução no diagnóstico da doença, causando impacto na vida dos pacientes.

Figura 1- Casos de hanseníase no Maranhão nos anos de 2018 a 2020.

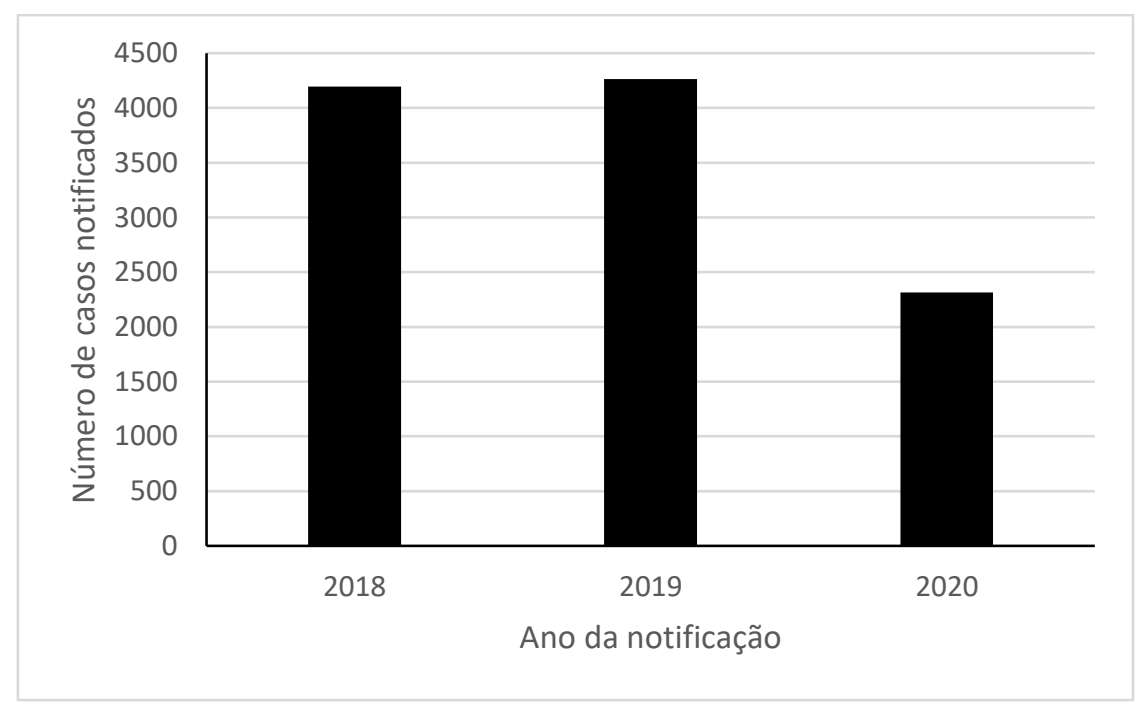

Fonte: Ministério da Saúde - Sistema de Informações de Agravos de Notificação do SUS (SINAN/SUS).

Quando se analisou a proporção de casos de Hanseníase segundo gênero, verificou-se a predominância do sexo masculino em todos os anos estudados, em 2018 com 57,6\%, 2019 com 59,9\% e 2020 com 59,8\% (Tabela 1). 
Tabela 1. Casos confirmados de Hanseníase segundo o sexo no Maranhão nos anos de 2018 a 2020.

\begin{tabular}{llcccc}
\hline & \multicolumn{2}{l}{ Masculino } & \multicolumn{2}{c}{ Feminino } & \multicolumn{2}{c}{ Total } \\
Ano de Notificação & $\mathrm{n}$ & $\%$ & $\mathrm{n}$ & $\%$ & $\mathrm{n}$ \\
\hline 2018 & 2.420 & 57,6 & 1.775 & 42,3 & 4.195 \\
2019 & 2.550 & 59,7 & 1.715 & 40,2 & 4.265 \\
2020 & 1.387 & 59,8 & 929 & 40,1 & 2.316 \\
\hline Total & 6.357 & & 4.419 & & 10.776 \\
\hline
\end{tabular}

Fonte: Ministério da Saúde/SVS - Sistema de Informação de Agravos de Notificação - SINAN.

Esses dados corroboram com o estudo de Anchieta et al. (2019) realizado no estado do Maranhão no período de 2001 a 2015 no qual dos 77.679 casos de hanseníase registrados, 57,7\% eram do sexo masculino. Este resultado mostra também uma similaridade com o estudo de, Monteiro et al. (2017) realizado no Nordeste brasileiro, resultado aponta que os homens têm maior contato social a ambientes de risco e menor preocupação com o corpo e estética quando comparado com mulheres, além da ausência deficiência de programas específicos voltados para esse grupo, o que pode garantir uma detecção precoce e prevenção para desenvolvimento de complicações mais graves.

Considerando as macrorregiões de saúde, a Norte concentrou o maior número de casos, em 20182,376 casos correspondendo a 53,63\%, 2019 com 2325 casos (54,51\%) e 2020 com 1345 casos $(58,07 \%)$ (Tabela 2).

Tabela 2. Casos confirmados de Hanseníase segundo a macrorregião de saúde no Maranhão nos anos de 2018 a 2020.

\begin{tabular}{|c|c|c|c|c|c|c|c|c|}
\hline \multirow[t]{2}{*}{$\begin{array}{l}\text { Ano } \\
\text { de notificação }\end{array}$} & \multicolumn{2}{|c|}{ Macrorregião sul } & \multicolumn{2}{|c|}{ Macrorregião norte } & \multicolumn{2}{|c|}{$\begin{array}{l}\text { Macrorregião } \\
\text { leste }\end{array}$} & \multicolumn{2}{|l|}{ Total } \\
\hline & $\mathrm{n}$ & $\%$ & $\mathrm{n}$ & $\%$ & $\mathrm{n}$ & $\%$ & $\mathrm{n}$ & $\%$ \\
\hline 2018 & 839 & 20 & 2.376 & 56,7 & 980 & 23,3 & 4.195 & \\
\hline 2019 & 901 & 21,1 & 2.353 & 55,16 & 1.039 & 24,3 & 4.265 & \\
\hline 2020 & 436 & 18,8 & 1.366 & 58,9 & 535 & 23,10 & 2.316 & \\
\hline Total & 2203 & & 6.095 & & 2.554 & & 10.776 & \\
\hline
\end{tabular}

Fonte: Ministério da Saúde/SVS - Sistema de Informação de Agravos de Notificação - SINAN.

Um estudo realizado por Ribeiro, Silva e Oliveira (2018), demonstrou que os coeficientes de prevalência da hanseníase no Brasil apresentam tendência de redução, porém observou-se que esse comportamento não foi visto em todas as regiões brasileiras, nas regiões Norte, Nordeste e Centro-Oeste ainda possuem um alto padrão de endemicidade.

Para Souza Júnior et al., (2020), o que pode justificar o fato do índice elevado de casos de hanseníase na Região Norte consequentemente no Maranhão, está relacionado a baixos valores no Índice de Desenvolvimento Humano (IDH), o que desafia o controle da doença, tornando assim a região endêmica.

Quanto à variável raça, a parda foi a mais prevalente dentre os anos estudados, seguida da raça parda e preta (Tabela $3)$. 
Tabela 3. Casos confirmados de Hanseníase segundo a raça no Maranhão nos anos de 2018 a 2020.

\begin{tabular}{|c|c|c|c|c|c|c|c|c|c|c|}
\hline \multirow[t]{2}{*}{ Ano } & \multicolumn{2}{|c|}{ Ig/Branco } & Branca & Preta & \multicolumn{2}{|c|}{ Amarela } & Parda & \multicolumn{2}{|c|}{ Indígena } & \multirow[t]{2}{*}{ Total } \\
\hline & $\mathrm{n}$ & $\%$ & $\%$ & $\%$ & $\mathrm{n}$ & $\%$ & $\%$ & $\mathrm{n}$ & $\%$ & \\
\hline 2018 & 64 & 1,5 & 57113,6 & 63915,2 & 47 & 1,12 & $2.86768,3$ & 7 & 0,16 & 4.195 \\
\hline 2019 & 62 & 1,4 & 55112,9 & 68716,1 & 39 & 0,91 & $2.90868,1$ & 18 & 0,42 & 4.265 \\
\hline 2020 & 47 & 2,0 & 32313,9 & 34414,8 & 13 & 0,56 & $1.58568,4$ & 4 & 0,17 & 2.316 \\
\hline Total & 173 & & 1.445 & 1.670 & 99 & & 7.360 & 29 & & 10.776 \\
\hline
\end{tabular}

Fonte: Ministério da Saúde/SVS - Sistema de Informação de Agravos de Notificação - SINAN.

Barbosa (2014) relata que esse achado reproduz um processo histórico de colonização, mistura de raças, migração de organização espacial no espaço urbano. Nas últimas décadas, especialmente devido às condições precárias de vida da população e ao acesso restrito aos bens e serviços coletivos, tais como escolas, serviços de educação e segurança de saúde, o processo de urbanização da hanseníase no Brasil se intensificou. Tais características se ligam ao espaço da cidade, onde o corpo urbano se fragiliza diante da elevada densidade populacional e vulnerabilidade socioeconômica, que determinam, em conjunto, um panorama geral de adoecimento e morte.

Quanto à escolaridade a maior ocorrência de hanseníase concentrou-se em indivíduos que frequentavam a $1^{\circ}$ a $4^{\mathrm{a}}$ série incompleta do ensino fundamental com (Tabela 4).

Segundo Azevedo (2018) quanto menor o grau de escolaridade, maior a probabilidade de ocorrência e reativação da hanseníase. Visto que, essa classe mostra pouco conhecimento e dificuldade de compreensão das orientações referentes ao tratamento, prevenção e autocuidado relacionado ao diagnóstico e entendimento e princípio da doença. Condições sociais, vulnerabilidade a respeito da educação, a hanseníase está associada a situações precárias de higiene, pobreza e baixo nível socioeconômico. 
Tabela 4. Casos confirmados de Hanseníase segundo a escolaridade no Maranhão nos anos de 2018 a 2020.

\begin{tabular}{lccc}
\hline Escolaridade & $\mathbf{2 0 1 8}$ & $\mathbf{2 0 1 9}$ & $\mathbf{2 0 2 0}$ \\
\hline Ig/Branco & 489 & 510 & 335 \\
Analfabeto & 581 & 588 & 290 \\
$1^{\circ}$ a $4^{\circ}$ série incompleta do EF & 928 & 841 & 448 \\
$4^{\circ}$ série completa do EF & 219 & 235 & 132 \\
$5^{\circ}$ a $8^{\circ}$ série incompleta & 690 & 641 & 314 \\
Ensino fundamental completo & 235 & 307 & 142 \\
Ensino médio incompleto & 257 & 307 & 142 \\
Ensino médio completo & 595 & 648 & 388 \\
Educação superior incompleta & 49 & 54 & 41 \\
Educação superior completa & 110 & 111 & 22 \\
\hline Não se aplica & 42 & 31 & 2.316 \\
\hline Total & 4.195 & 4.265 & 22 \\
\hline
\end{tabular}

Fonte: Ministério da Saúde/SVS - Sistema de Informação de Agravos de Notificação - SINAN.

Quanto à forma clínica, de acordo com a Tabela 5, observa-se que a forma clínica mais prevalente foi a dimorfa, seguido da forma Virchowiana.

Tabela 5. Casos confirmados de Hanseníase segundo a forma clínica no Maranhão nos anos de 2018 a 2020.

\begin{tabular}{|c|c|c|c|c|c|c|c|c|c|c|c|c|c|}
\hline \multirow{2}{*}{$\begin{array}{c}\text { Ano de } \\
\text { notificação }\end{array}$} & \multicolumn{2}{|c|}{ Ign } & \multicolumn{2}{|c|}{ Ind } & \multicolumn{2}{|c|}{$\mathbf{T b}$} & \multicolumn{2}{|c|}{ Dm } & \multicolumn{2}{|c|}{ Vw } & \multicolumn{2}{|c|}{$\mathrm{Nc}$} & Total \\
\hline & $\mathrm{n}$ & $\%$ & $\mathrm{n}$ & $\%$ & $\mathrm{n}$ & $\%$ & $\mathrm{n}$ & $\%$ & $\mathrm{n}$ & $\%$ & $\mathrm{n}$ & $\%$ & $\%$ \\
\hline 2018 & 113 & 2,69 & 401 & 9,55 & 451 & 10,75 & 2.355 & 56,13 & 702 & 16,73 & 173 & 4,12 & 4.195 \\
\hline 2019 & 135 & 3,16 & 401 & 9,40 & 413 & 9,68 & 2.375 & 55,68 & 745 & 17,46 & 196 & 4,59 & 4.265 \\
\hline 2020 & 87 & 3,75 & 194 & 8,37 & 219 & 9,45 & 1.271 & 54,87 & 413 & 17,83 & 132 & 5,69 & 2.316 \\
\hline Total & \multicolumn{2}{|c|}{335} & \multicolumn{2}{|c|}{996} & \multicolumn{2}{|c|}{1.083} & \multicolumn{2}{|c|}{6.001} & \multicolumn{2}{|c|}{1.860} & \multicolumn{2}{|c|}{501} & 10.776 \\
\hline
\end{tabular}

Legenda: Ign - ignorado e branco; Ind - indeterminada; Tb- tuberculoide; Dm - dimorfa, Vw- virchowiana; Nc - não classificada. Fonte: Ministério da Saúde/SVS - Sistema de Informação de Agravos de Notificação - SINAN.

Para Silva et al. (2020), o alto índice de pacientes dimorfos e virchowianos em conjunto com o baixo número de casos de hanseníase indeterminada e tuberculóide, deixa a sugerir diagnósticos tardios, uma vez que a maioria dos casos notificados da doença, ela já se encontra em estágio avançado. Os estágios dimorfos e virchowianos são as formas mais contagiosas da hanseníase, quando a hanseníase se encontra nesse estágio, podem ocorrer deformidades levando a incapacidade física, além de 
continuarem com ciclo de transmissão do bacilo de Hansen. O diagnóstico da doença neste estágio implica ser um diagnóstico tardio.

\section{Considerações Finais}

No presente estudo foi observado que a hanseníase foi predominante no sexo masculino, na macrorregião Norte, nas pessoas da raça parda, em pessoas com baixo grau de escolaridade e a forma clínica mais comum foi a dimorfa.

As condições sociais e vulnerabilidade associam a hanseníase a situações precárias de higiene e baixo nível econômico, levando a dificuldade de acesso à saúde. Espera-se, portanto, que os órgãos estaduais e municipais de saúde, promovam ao acesso a informações de saúde para população afetada, ações que promovam melhora dos indicadores de controle da doença no Maranhão, visando aperfeiçoar os aspectos relacionados à educação e saúde, tendo maior atenção ao público masculino, para que o tratamento da doença ocorra de forma eficiente.

\section{Referências}

Almeida, F. A. F. L., \& Milan, G. (2020). Diagnóstico de hanseníase em Porto Nacional/TO no período de 2013 a 2017. Scire Salutis, 10(3), 104-112.

Anchieta, J. D. J. S., Costa, L. M. M. da, Campos, L. C., Vieira, M. D. R., Mota, O. S., Morais Neto, O. L., Souza, M. R. de, \& Guimarães, R. A. (2019). Trend analysis of leprosy indicators in a hyperendemic Brazilian state, 2001-2015. Revista de Saúde Pública, 53, 61.

Araújo, K. M. D. F. A. (2018). Epidemiologia da Hanseníase na Paraíba e sua relação com a cobertura da Atenção Primária à Saúde e condições socioeconômicas. Tese de doutorado da Escola de Enfermagem da Universidade Federal de Minas Gerais.

Azevedo, K. F. D. (2018). Caracterização clínica epidemiológica dos pacientes com hanseníase acometidos por incapacidade física no nordeste brasileiro. Trabalho de Conclusão de Curso (TCC) do Curso de Bacharelado em Enfermagem da Universidade Federal de Campina Grande.

Barbosa, D. R., Almeida, M. G., \& dos Santos, A. G. (2014). Características epidemiológicas e espaciais da hanseníase no Estado do Maranhão, Brasil, 20012012. Medicina (Ribeirao Preto Online), 47(4), 347-356.

da Silva, P. S. R., Cunha, N. G. T., Oliveira, L. S., \& Santos, M. C. A. (2020). Perfil clínico-epidemiológico de pacientes portadores de hanseníase em um município do Maranhão. Revista Eletrônica Acervo Saúde, 12(8), e3468-e3468.

Carvalho, A. V., Fonseca, F. C. G., Fernandes, N. A. F., da Mata, T. S., \& Lira, J. J. Hanseníase: tratamento e prevalência das sequelas neuromotoras. Revista Saberes da Faculdade São Paulo.

Felipe, C. O., de Medeiros, A. C. T. R., de Queiroz, M. V. R., Vallandro, E. I. D., \& de Abreu Sarmenghi, K. D. (2021). Impactos do COVID-19 no ambulatório e residência médica em dermatologia. Revista Científica da Faculdade de Medicina de Campos, 16(1), 42-45.

Façanha, A. T. F., da Conceição, H. N., Oliveira, M. R., Borges, L. V. A., Pereira, B. M., Moura, L. R. P., ... \& Câmara, J. T. (2020). Análise das incapacidades físicas por hanseníase em uma cidade do interior do Maranhão, Brasil. Research, Society and Development, 9(2), e75922055-e75922055.

Gomes, T. C. F. (2018). Impacto na vida social do portador de hanseníase com reações hansênicas atendidos em um ambulatório de referência em São Luís-MA.

Grossi, M. A. D. F., Leboeuf, M. A. A., Andrade, A. R. C. D., Lyon, S., Antunes, C. M. D. F., \& Bührer-Sékula, S. (2008). A influência do teste sorológico ML Flow na classificação da hanseníase. Revista da Sociedade Brasileira de Medicina Tropical, 41, 34-38.

Lopes, F. D. C., Ramos, A. C. V., Pascoal, L. M., Santos, F. S., Rolim, I. L. T. P., Serra, M. A. A. D. O., ... \& Santos, M. (2021). Hanseníase no contexto da Estratégia Saúde da Família em cenário endêmico do Maranhão: prevalência e fatores associados. Ciência \& Saúde Coletiva, 26, $1805-1816$.

Mendonça, C. A. D. S. (2018). Perfil clínico-epidemiológico dos pacientes diagnosticados com hanseníase no estado do Maranhão de 2006 a 2015. Trabalho de Conclusão de Curso (TCC) do Curso de Bacharelado em Enfermagem da Universidade Federal do Maranhão.

Ribeiro, M. D. A., Silva, J. C. A., \& Oliveira, S. B. (2018). Estudo epidemiológico da hanseníase no Brasil: reflexão sobre as metas de eliminação. Revista Panamericana de Salud Pública, 42, e42.

Pacheco, M. A. B., Aires, M. L. L., \& Seixas, E. S. (2014). Prevalência e controle de hanseníase: pesquisa em uma ocupação urbana de São Luís, Maranhão, Brasil. Revista Brasileira de Medicina de Família e Comunidade, 9(30), 23-30.

Rocha, A. D. J. (2012). O impacto social das doenças negligenciadas no Brasil e no mundo.

Santos, G. R. B., Aragão, F. B. A., da Silva Brasil, G. V., da Silva, R. L., Junior, A. R. G., de Andrade, L. M. R. L., ... \& Batista, J. E. (2018). Prevalência de hanseníase em São Luís-Maranhão entre os anos de 2013 a 2015. Journal of Nursing and Health, 8(2).

Sousa, F. D. C. A., Soares, H. V. A., Lemos, L. E. A. S., Reis, D. M., da Silva, W. C., \& de Sousa Rodrigues, L. A. (2020). Perfil epidemiológico de doenças negligenciadas de notificação compulsória no Brasil com análise dos investimentos governamentais nessa área. Research, Society and Development, 9(1), e62911610-e62911610. 